\title{
How digital asset management improved the production of packaging artwork for Philips DAP
}

\section{Geert Wirtjes}

is External Program Manager at Philips Domestic Appliances, and has been responsible for the translation of business requirements from within the marketing communications area into an effective digital asset management solution for Philips DAP.

\section{Debbie Wirtjes-Lewis}

is Director of Corporate Communications, Wirtjes Management BV. She has more than 14 years' experience in the area of communications, working as a broadcast journalist and as the Executive Director of the American Chamber of Commerce of Trinidad and Tobago. Debbie currently trains executives in the art of making corporate presentations and dealing with the media. She also provides editorial and advisory services for corporate presentations.

Keywords: performance management, packaging artwork production, process redesign, digital asset management, workflow automation, collaboration

Abstract Since the introduction of a digital asset management (DAM) solution two years ago, Philips Domestic Appliances and Personal Care has been able to realise a cost reduction of 35 per cent project in the area of packaging artwork production. The traffic department has increased its ability to handle projects by 200 per cent. In general, the organization was able to reduce the cost of distributing assets by 85 per cent, while the number of assets being reused rose with a factor of 1,000 .

This paper first identifies some key problem areas related to packaging artwork production, focusing on relationships between operational parties within the 'graphical supply chain'. It highlights the common problems that add to the cost of failure and illustrates step by step how redesigning the supply chain using DAM technology and integrated workflow management could eliminate errors and so improve overall performance.

Management,

PO Box 77900

1070 MX Amsterdam

The Netherlands

Tel: +31655191897

Email: geert.wirtjes@

philips.com

\section{INTRODUCTION}

This paper provides an overview of the essential elements of performance management in the area of packaging artwork production. It also highlights the improvements made to business processes after having established a framework for performance management.

The first part will describe some characteristics of a "graphical supply chain" related to artwork production in general. Exploring relationships between operational parties in the artwork supply chain for packaging will reveal some common problems and their negative impacts on business performance.

The second part will describe proposed changes in processes and ways of working, and ultimately the benefits and challenges derived from introducing digital asset management and related technologies in this particular business environment.

\section{THE GRAPHICAL SUPPLY CHAIN}

Continuous pressures in the marketplace require enterprises to increase agility in the marketing communications area. Multinational business-toconsumer oriented enterprises face tough challenges rolling-out global marketing campaigns, while trying to increase customerfocus and customer-centric delivery at a local level in order to create maximum effectiveness at point of purchase.

A typical overview of the scope and variety of the parties involved in the process of artwork creation and production is depicted in Figure 1. ${ }^{1}$

The setup as described in the figure clearly demonstrates the complexity in a graphical supply chain from an overall project management perspective. Every time the number of contributors doubles, the number of opportunities to miss vital deadlines is squared, and delivering artwork in a predictable, 


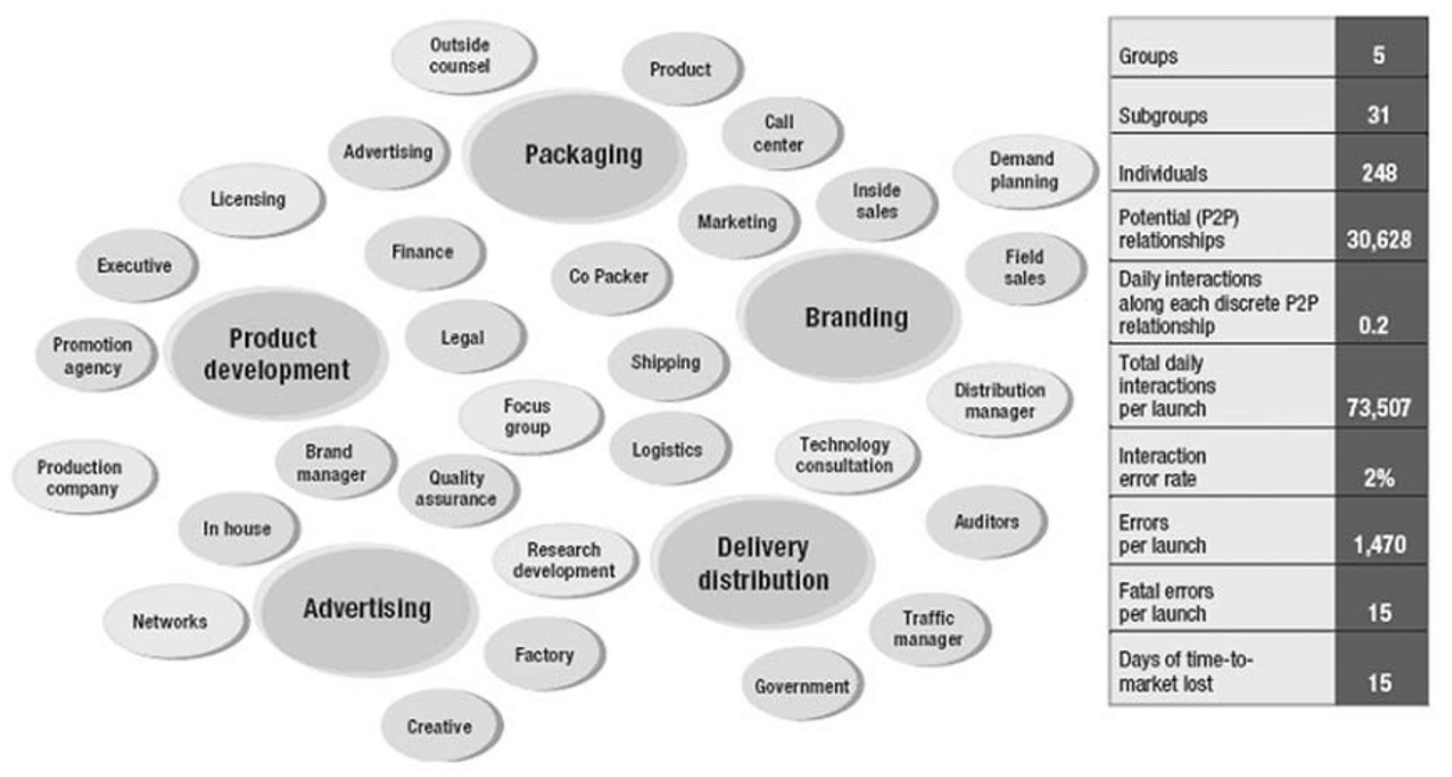

Figure 1: Artwork creation and production in the graphical supply chain Source: (C) GISTICS 2003. All rights reserved.

trustworthy and timely manner is under tremendous pressure.

In a multinational environment with centralized creation of packaging artwork that needs to be localized for over 100 countries in more than 45 languages, errors in the later stages of the value chain have devastating impact on throughput time, actual costs and delivery reliability. Even when calculations are done with a relatively low "interaction error rate," the cost of failure could be astonishing, caused by a high number of approval cycles and a high volume of potentially unnecessary rework.

With the emergence of digital asset management (DAM) technology around 2001, came the challenge of converting traditional ways of working into a " 100 per cent digital workflow." This affects the very nature of the artwork objects (digital assets) themselves, the nature of tasks performed, as well as the ability to generate real-time management information as a basis for continuous process improvement and the overall governance model. The continuous pressure on agility and performance as previously mentioned, and by its own nature, the technically and organizationally challenging area of expertise, combined with the recent introduction of DAM applications in the marketplace make this field a rewarding area for improvement.
The rest of this paper describes some recent improvement measures in the area of packaging artwork production for Philips Domestic Appliances, directly related to DAM technology and integrated workflow management functionality.

\section{SUPPLY CHAIN REDESIGN}

Figure 2 shows the Capabilities Maturity Model as a guide for implementing IT-oriented project management. Enterprises using this model can assess the maturity level at which project management processes are practiced within their own environment. Level 0 represents low maturity, and level 5 best-practice high maturity.

At Philips DAP, this model has been used as a reference to describe the maturity levels of project management skills of all parties within the graphical supply chain. Although maturity levels varied at the start of the project in 2001, both internal and external critical workgroups did not generally surpass level 1 .

Ad hoc project management contributed to an increase in necessary artwork amendments in the later stages of artwork completion, which clearly accounted for a surprisingly large sum of cost of failure. The relationship between amendments in time and total cost of failure is described in Figure 3. 


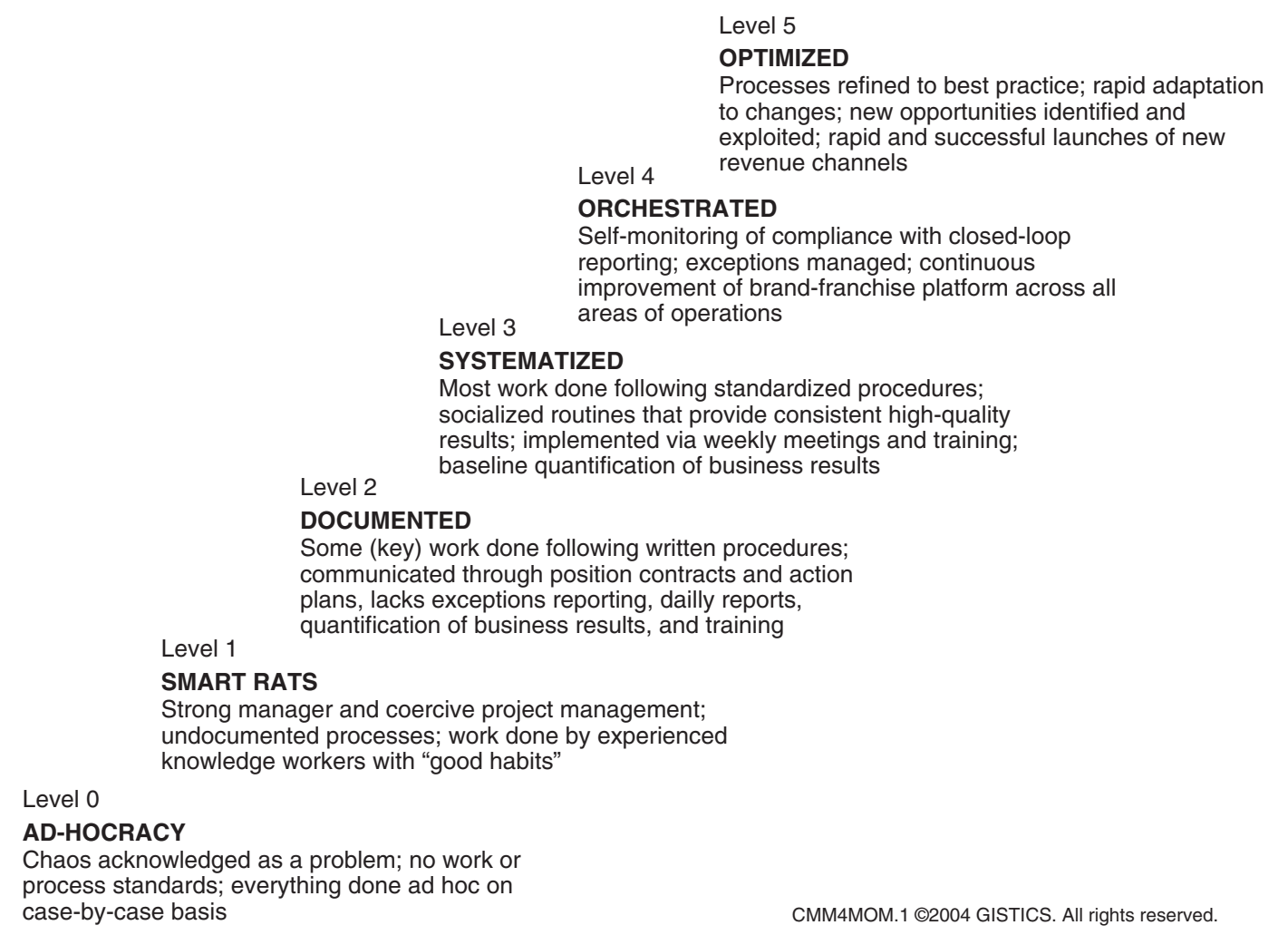

Figure 2: Capabilities maturity model

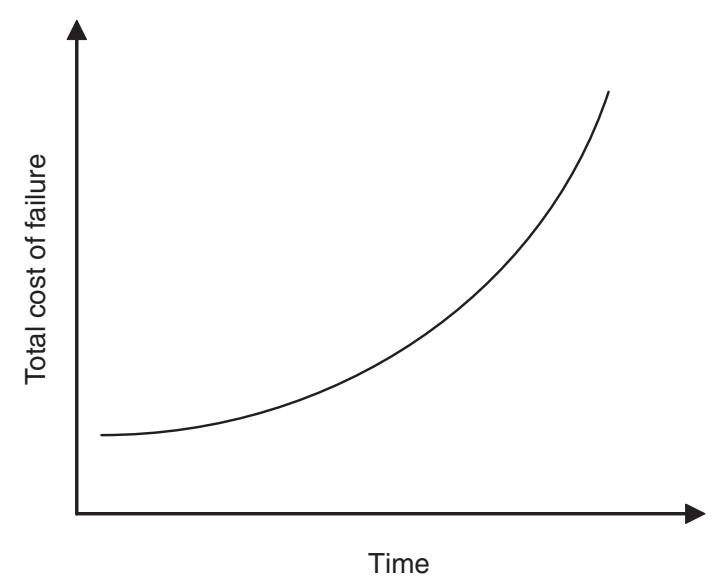

Figure 3: Cost of failure associated with amendments in different stages of artwork completion

Based on this analysis a central improvement team was set up to drive improvements.

Relationships in the supply chain and the role of partnerships were reevaluated.

\section{The driving force}

An integral perspective on the problems encountered in the supply chain was clearly needed. Rather than trying to solve individual problems with individual stakeholders, the focus was on finding relationships between amendments in later stages of artwork completions traced back to root causes in earlier stages in the supply chain.

The analysis was done through the traffic department whose employees quickly learned that problems encountered in the later stages of the supply chain could be effectively addressed by modifications in their work methods in the earlier stages. By shifting their management style and formally taking ownership of (integral) project management, this department became a competence centre in the entire chain.

The internal goal of the traffic department was to elevate their project management skills to level 3 of the Capabilities Maturity Model within the shortest possible timeframe and serve as a best-practice model for other parties. The 
intention was to actively drive a capabilities maturity methodology into the partnerbusinesses and provide a common collaboration sharing network for all parties involved.

Level 3 was described as follows.

- A graphical production environment where standard processes for creating artwork should be documented and aligned with the global rollout of a campaign.

- Processes described should be used to help members within the packaging value chain to perform more effectively.

- Roles and responsibilities of all stakeholders should be clearly defined and understood. Management should have up-to-date insight into the progress of all packaging artwork related projects.

- Project cost, schedule, and requirements should be under control, and product quality should be tracked.

This was accomplished by analyzing the internal work methods, optimizing these methods to serve as a benchmark and then prototyping the usage through a simple MS Access application before implementing a full-fledged workflow management system.

\section{Integral management}

To address root causes in the graphical supply chain effectively, however, traffic management needed to start managing beyond their departmental boundaries. This required a detailed understanding of how technology was operating and a more thorough understanding of the print supply chain. To facilitate this, meetings were set up between print suppliers, lithographers, designers, product management and traffic management. At these meetings, new package briefings and conceptual designs were discussed against workflow diagrams comprising three levels:

— the artwork itself (ie digital assets): projected from initial brief through production to the delivered approved file to the designated printer or factory anywhere in the world;

- the activities and tasks performed in the various stages, defining their owners and throughput time and cost;
- the role of management information and critical path control.

Generally speaking these sessions made parties down the chain (eg design) more aware of the total cost associated with the execution of a design, as well as technical problems arising at factory level at time of mass production. Parties up-stream became more receptive to a

marketing approach and the need for innovative packaging concepts.

On the artwork level the most recurring issues with highest financial impact were traced back to:

- the complexity of version control;

- highly limited reuse of assets;

— a large number of "comfort" proofs, eg expensive proofing methods used for other purposes than color management or print issues.

Main issues at the level of activities arose from the fact that a standard process for artwork production was not in place. Knowledge workers generally had no upfront knowledge of the time and money available for performing their task, nor were there any predefined limits of acceptable number of approval cycles. Furthermore, paper-based approval cycles were based on a linear process. Time needed for worldwide physical distribution of proofs and subsequent delays at the desk of the approvers also added to throughput time.

The lack of standardization and workflow transparency, combined with the fact that missing or non-approved data at crucial stages, caused artwork to be driven through the chain staying "conceptual."

What was needed was a model clearly describing the desired state of artwork based on clear stage gating.

Finally, ad hoc processes did not deliver the data required to manage time and money integrally. Bringing production parties together revealed the need for one data model fed by data from individual work processes to create transparency and predictability.

\section{Managing strategic partnerships}

A profile for preferred suppliers was created, based on which current suppliers were (re) evaluated against the following criteria: 
- the total transaction cost of a strategic partner should be sufficiently lower compared with similar activities handled internally;

- a strategic partner should be chosen based on the strength of their balance sheet, they should be able to demonstrate benchmarked best practices, invest heavily in workflow automation and employ a set of standards for governance;

- their data systems should be able to produce and send data into a project management portal thereby supporting the performance dashboard of the traffic management department.

After being armed with a thorough understanding of the graphical supply chain, an analysis of the main drivers causing cost of failure and an ideal model for an integral management approach, technologies were then implemented to reduce throughput time, improve cost efficiency and enhance the quality of the processes. This resulted in improved delivery reliability.

\section{IMPLEMENTED TECHNOLOGY}

So how exactly did asset management technology support this new outlook on integral project management in the value chain? How exactly did it contribute to either speeding up the process by replacing repetitive manual tasks by automation; reducing the transaction cost of tasks and activities, and/or improve the quality (brand consistency) of the artwork?

As a general rule, by installing one central repository for all assets produced for packaging artwork, Philips DAP (re)gained control over company assets and subsequently became more independent of its suppliers.

By automating standardized workflows and deploying those workflows through a common platform for collaboration, Philips DAP was able to drive improvements in project management to its stakeholders' business while having a transparent outlook over the entire chain.

With the basics of DAM and workflow tools in place, existing applications supporting online proofing and real-time reporting were then integrated into a custom-made solution for the packaging supply chain.

\section{DAM}

As previously described, two key drivers for cost inefficiencies were lack of version control and limited reuse of assets. Implementation of a DAM system enabled Philips DAP to regain control over its assets by centrally storing all its assets into one database. The application was set up to keep track of:

_ “revisions of final form artwork," eg versions of Quark Xpress or ArtPro files arising from amendments in artwork;

_ "variants of assets" arising from the fact that files were used for a different purpose than originally intended;

_ "sub-assets" being the individual elements used in final form artwork eg Tiff, EPS etc.

By working with multiple agencies with different levels of automation, these relationships between assets could be determined either manually or automatically upon ingestion of the artwork in the database.

The distribution of assets became faster and above all cheaper because they were now able to be unlocked through the use of an internet browser. Tracking and tracing the (re)usage of assets over a four-year period (see Figure 4) showed a steady growth of assets being (re)used for different purposes. There was also a sharp decline in the associated costs of distributing those assets through the organization.

Far more important than a payback time of less than 1.5 years, based on a reduction of the cost of failure and the reduced cost of distribution, was that the introduction of DAM

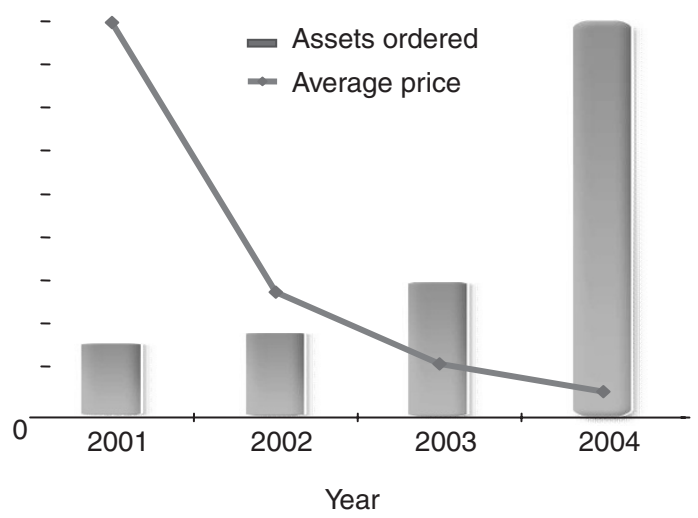

Figure 4: Sustainable cost efficiency: Reduced cost of distribution and increased number of centrally managed assets 
had led to a significant change in work methods and a sustainable cost efficiency.

\section{Workflow management and collaboration}

\section{Workflow management}

The analysis of the tasks carried out by parties in the value chain led to the definition of five types of projects. For each project type a standardized flow of activities was described. A workflow application was developed, allowing projects to be generated based on XML templates that described the activity flows. Each activity was then assigned a formal owner, a budget and a maximum allowable duration. Furthermore, the necessary information to carry out a specific activity was defined in standardized briefings associated with an activity. Formal "checkpoints" per activity were incorporated into the application, leaving the owner only three choices:

- continuing work based on complete and accurate briefing/information;

- continuing work explicitly acknowledging lack of completeness thereby formally taking the responsibility for potential (financial) issues at later stages in the project, however, not delaying the process at that particular stage;

- declining work based on incomplete data, most likely delaying the process, however addressing problems in the earliest stages, eventually driving down total cost of failure.

All costs involved in creating a piece of artwork were directly charged to the associated activity, making it possible to compare budgeted figures with actual spend. Log files were generated by the automated workflow, enabling operations management to confront knowledge workers with their decisions and the financial impact of these decisions.

\section{Collaboration}

A third-party application called RealTimeProof (recently bought by Eastman Kodak Company) was integrated with the workflow management application. RealTimeProof enabled all workflow partners to collaborate in real time on original, full-resolution production files. By unlocking production files over the internet, formally registering comments and approvals and changing linear approval cycles into parallel ones, impressive reductions in throughput times were realized. Furthermore, a worldwide network of inkjet printers was rolled-out, enabling partners to "hard proof' artwork directly from the workflow application.

\section{Online reporting}

Activity-based data derived from automated workflows were processed in real time and published in meaningful reports on the internet for a variety of stakeholders. In order to drive down transaction costs in the supply chain, while improving the quality of the output, reports and key performance indicators were calculated, measuring among other things:

- delivery of cost reduction and value added by strategic partners;

- delivery of "first time right" principle;

- the number of amendments that are made to artworks and imagery:

- by type/cost;

- by individual;

— the split of work by activity with financials analyzed;

- the number of days on approval;

- the number of approval cycles;

- the number of corrections;

- the number of projects handled per project type;

- duration of tasks and activities against their norm;

- the compliance with delivery dates of final form artwork.

\section{RESULTS}

Apart from an overall increase in the understanding between parties in the packaging supply chain, improved communication and a feeling of partnership, the impact of process redesign supported by DAM and related technologies has been significant. Improved version control reduced cost of failure. The cost of distributing assets through the organization was reduced by 85 per cent, while the number of assets being reused rose with a factor of 1,000 .

In less than two years, the improvement efforts in the packaging area delivered a sustainable cost reduction on average of 35 per 
cent per project and a reduction in throughput time of 60 per cent. With the same number of employees, the traffic department was able to handle an increase in the number of projects of over 200 per cent in three years. A result that would have been impossible without the implementation of DAM combined with standardized, automated workflows.

\section{Reference}

1 Moon, M. (2003) GISTICS. 